\title{
ON SOME FIXED POINTS THEOREMS IN GENERALIZED COMPLETE METRIC SPACES
}

\author{
BY BEATRIZ MARGOLIS ${ }^{1}$
}

Communicated by J. B. Diaz, September 21, 1967

In Theorem 2 of $[1]^{2} \mathrm{~A}$. F. Monna generalized a result by W. A. J. Luxemburg on fixed points [2], valid for one operator in a generalized complete metric space, to a suitable family of operators; this result was later completed by M. Edelstein [3].

Clearly, when the family reduces to a unique element (i.e. $T_{i} \equiv T$ for all $i$ ), one gets Luxemburg's result. But if one considers the family of iterates of $T: T_{i}=T^{i}(i=1,2, \cdots)$, since Hypothesis 1 of Monna's Theorem requires $d\left(T_{i} x, T_{i} y\right) \leqq \rho d(x, y)(i=1,2, \cdots)$ when $d(x, y)$ $\leqq C$, we must have, in particular, $d(T x, T y) \leqq \rho d(x, y)$, and Luxemburg's Theorem applies, providing even with a stronger conclusion than Monna's for this particular situation. In order to include this case as a strict generalization of Luxemburg's result, we relax Hypothesis 1 slightly, thus including also Monna's Theorem, and at the same time we get for the family $\left\{T^{i}\right\}$ a nontrivial result. This last assertion will be clarified with an example. This constitutes $\$ 1$ of our paper.

In $\$ 2$ we give some fixed point results for a family of operators with $\rho=1$.

1. Theorem 1. Let $(X, d)$ be a generalized complete metric space, ${ }^{3}$ and $\left\{T_{i}\right\}_{i=1,2,} \ldots$ a family of self-mappings of $X$, closed under composition, such that

(1) There exist constants $C>0,0 \leqq \rho<1$, and an integer $m \geqq 1$ such that if $x, y \in X$ and $d(x, y) \leqq C$, then

$$
d\left(T_{m+k} x, T_{m+k} y\right) \leqq \rho d(x, y) ; \quad k=0,1,2, \cdots .
$$

(2) $T_{i}=T_{j}=T_{j} T_{i} ; i, j=1,2, \cdots$

(3) Let $x_{0} \in X$ be arbitrary, and define $x_{n}=T_{n} x_{n-1}(n=1,2, \cdots)$. Then there exists $N\left(x_{0}\right)$ such that $d\left(T_{n+k} x_{n}, x_{n}\right) \leqq C$, for $n \geqq N$, $k=1,2 \cdots$.

Then, there exists a $\xi \in X$ such that $x_{n} \rightarrow \xi$ and $T_{n} \xi \rightarrow \xi$ as $n \rightarrow \infty$.

Furthermore, (if)

${ }^{1}$ Research sponsored by Fundación Bariloche, República Argentina. The author is now at Universidad Nacional de La Plata, República Argentina.

2 Numbers in [ ] correspond to References.

8 We follow Luxemburg's denomination. 
(4) $T_{n} x \rightarrow x, T_{n} y \rightarrow y$ as $n \rightarrow \infty \Rightarrow d(x, y) \leqq C$ then $\xi$ is unique, and $T_{h+k} \xi=\xi(h=\max (m, N) ; k=0,1, \cdots)$.

(In each case, convergence refers to convergence in the metric $d$. )

REMARKs. (a) When $m=1$, we have Monna's Theorem, with Edelstein's completion of it.

(b) Example 1 will show that our Hypothesis 1 is strictly more general than Monna's.

(c) If $T_{i}=\widetilde{T}^{i}(i=1,2, \cdots)$ the conclusion means that $\xi$ is periodic under $\tilde{T}$ with period not greater than $\max (m, N)$.

(d) Since $\xi$ is unique, and $T_{k} \xi=T_{k} T_{h} \xi=T_{h} T_{k} \xi$, we have $T_{k} \xi=\xi$, and $\xi$ is actually a fixed point of all $T_{k}(k=1,2, \cdots)$.

Proof of Theorem 1. It will not be given in detail, since it follows mainly the procedures used by Luxemburg and Monna.

Let $x_{0} \in X$. Then, in the usual manner, it can be shown that for $n \geqq N+m: d\left(x_{n+h}, x_{n}\right) \leqq \rho^{n-N-m} C(1-\rho)^{-1}$, and all results follow like in Luxemburg, Monna and Edelstein.

We observe, letting $h \rightarrow \infty$, that the last inequality gives an estimate of the rate of convergence of the sequence $\left\{x_{n}\right\}$, namely

$$
d\left(x_{n}, \xi\right) \leqq \rho^{n-N-m} C(1-\rho)^{-1} .
$$

Example 1. Let $X=\left\{x=e^{i \theta} / 0 \leqq \theta \leqq \pi / 2\right\}$ and, for $x, y \in X$, define

$$
\begin{aligned}
d(x, y) & =0, \quad \text { if } \theta_{x}=\theta_{y}=\pi / 2, \\
& =\left|\operatorname{tg} \theta_{x}-\operatorname{tg} \theta_{y}\right|, \quad \text { otherwise. }
\end{aligned}
$$

Clearly, $d(x, y)=0$ iff $x=y, d(x, y)=d(y, x), d(x, y) \leqq d(x, z)$ $+d(y, z)$, for $x, y, z \in X$.

Assume $\left\{x_{n}=e^{i \theta_{n}}\right\}$ is a Cauchy sequence in $(X, d)$. Since, for $m, n \geqq n_{0}, d\left(x_{m}, x_{n}\right)$ is bounded, we have that either $\theta_{n}=\theta=\pi / 2$ for $n \geqq n_{0}$, or $0 \leqq \theta_{n}<\pi / 2-\epsilon$ for some $\epsilon>0, n \geqq n_{0}$. In the last case, since

$$
\begin{aligned}
d\left(x_{m}, x_{n}\right) & =\left|\operatorname{tg} \theta_{n}-\operatorname{tg} \theta_{m}\right|=\left|\operatorname{tg}\left(\theta_{n}-\theta_{m}\right)\right|\left|1+\operatorname{tg} \theta_{n} \cdot \operatorname{tg} \theta_{m}\right| \\
& \geqq\left|\operatorname{tg}\left(\theta_{n}-\theta_{m}\right)\right|,
\end{aligned}
$$

we have that $\left\{\theta_{n}\right\}$ is a Cauchy sequence in $[0, \pi / 2-\epsilon]$; hence, $\theta_{n} \rightarrow \theta$ as $n \rightarrow \infty$. Let $x=e^{i \theta}$. In any case, $d\left(x_{n}, x\right) \rightarrow 0$ as $n \rightarrow \infty$, and $(X, d)$ is a generalized complete metric space.

Define $T: X \rightarrow X$ as follows

$$
\begin{aligned}
T x & =e^{i 0}, & 0 & \leqq \theta<\pi / 8, \\
& =e^{i(\theta+\pi / 8)}, & \pi / 8 & \leqq \theta<3 \pi / 8, \\
& =e^{i(\theta-3 \pi / 8)}, & 3 \pi / 8 & \leqq \theta<\pi / 2, \\
& =e^{i 0}, & \theta & =\pi / 2 .
\end{aligned}
$$


Then

$$
\begin{array}{rlrl}
T^{2} x & =e^{i 0}, & 0 \leqq \theta & <\pi / 8, \\
& =e^{i(\theta+\pi / 4)}, & \pi / 8 \leqq \theta<\pi / 4, \\
& =e^{i(\theta-\pi / 4)}, & \pi / 4 \leqq \theta<3 \pi / 8, \\
& =e^{i 0}, & & 3 \pi / 8 \leqq \theta \leqq \pi / 2 . \\
T^{3} x & =e^{i \theta}, & & 0 \leqq \theta<\pi / 8, \\
& =e^{i(\theta-\pi / 8)}, & \pi / 8 \leqq \theta<\pi / 4, \\
& =e^{i 0}, & \pi / 4 \leqq \theta \leqq \pi / 2, \\
T^{4} x & =e^{i 0} & & 0 \leqq \theta \leqq \pi / 2 .
\end{array}
$$

Hence, neither $T$ nor $T^{2}$ nor $T^{3}$ satisfy Hypothesis 1 of Theorem 1 , for they are not continuous. Obviously, $T^{4}$ satisfies this condition with arbitrary $C>0$ and arbitrary $\rho, 0 \leqq \rho<1$, so that in this case $m=4$.

As for Hypothesis 3 of the Theorem, let $x_{0}=e^{i \theta_{0}}$. It is not difficult to see that if, for instance, $C=1$, and:

(a) $0 \leqq \theta_{0}<\pi / 8$, then $N\left(x_{0}\right)=0$.

(b) $\pi / 8 \leqq \theta_{0}<3 \pi / 8$, then $N\left(x_{0}\right)=2$.

(c) $3 \pi / 8 \leqq \theta_{0} \leqq \pi / 2$, then $N\left(x_{0}\right)=1$.

Condition 4 clearly holds.

Also, Remark (d) implies that $T x_{0}=x_{0}$ iff $x_{0}=e^{i \theta}$.

2. Our aim now is to obtain similar results for a family of operators satisfying Hypothesis 1 of Theorem 1 with a strict inequality sign and $\rho=1$. We will have to add some further requirements, for even with $T_{i} \equiv T$ the theorem would not hold. (See, for instance, [4, Remark 2].)

ThEOREM 2. Let $(X, d)$ be a generalized complete metric space, and $\left\{T_{i}\right\}_{i=1,2, \ldots}$ a family of self-mappings of $X$, such that

(1) There exist a constant $C>0$ and an integer $m \geqq 1$ such that if $x, y \in X$ and $0<d(x, y) \leqq C$ then $d\left(T_{m+k} x, T_{m+k} y\right)<d(x, y) ; k=0,1, \cdots$.

(2) $T_{i} T_{j}=T_{j} T_{i} ; i, j=1,2, \cdots$

(3) Let $x_{0} \in X$ be arbitrary, and define $x_{n}=T_{n} x_{n-1}(n=1,2, \cdots)$.

Then there exists $N\left(x_{0}\right)$ such that

(a) $d\left(T_{n+k} x_{n}, x_{n}\right) \leqq C ; n \geqq N, k=1,2, \cdots$,

$$
\frac{d\left(T_{n+k+1} x_{n+i+2}, x_{n+i+2}\right)}{d\left(T_{n+k+1} x_{n+i+1}, x_{n+i+1}\right)} \leqq \frac{d\left(T_{n+k+1} x_{n+i+1}, x_{n+i+1}\right)}{d\left(T_{n+k+1} x_{n+i}, x_{n+i}\right)},
$$


whenever the denominators do not vanish and $n \geqq N, k=1,2, \cdots$; $0 \leqq i \leqq k-1$.

(c) $\frac{d\left(T_{n+k+1} x_{n+1}, x_{n+1}\right)}{d\left(T_{n+k+1} x_{n}, x_{n}\right)} \leqq \frac{d\left(T_{n+k} x_{n+1}, x_{n+1}\right)}{d\left(T_{n+k} x_{n}, x_{n}\right)} ; \quad k=1,2, \cdots$,

whenever the denominators do not vanish.

(4) $T_{p} x=x, T_{p} y=y, p \geqq m \Rightarrow d(x, y) \leqq C$.

Then, there exists $a \xi \in X$ such that $x_{n} \rightarrow \xi$, and $T_{n} \xi \rightarrow \xi$ as $n \rightarrow \infty$.

Furthermore, if

(5) For some integer $h \geqq m: T_{h} T_{i}=T_{h+i}(i=1,2, \cdots)$ (and hence $\left\{T_{i}\right\}$ is a finitely generated semigroup), then $\xi$ is unique, and $T_{i} \xi=\xi$; $i=1,2, \cdots$.

(In each case, convergence refers to convergence in the metric d.) REMARKs. (a) When $T_{i} \equiv T$, condition (3c) is obviously satisfied. Moreover, in this case, although (5) implies the trivial transformation $T x=\xi, \forall x \in X$, we get the whole conclusion without it.

(b) When $T_{i}=\widetilde{T}^{i}$, (5) holds for any integer $h$, and hence $\xi$ is fixed under $\widetilde{T}$.

Before proving the Theorem, we need the following

LеммA. If for some integer $h \geqq m$, and some integer $p$, we have $T_{h} x_{p}=x_{p}$, then $x_{p}=x_{p+1}=x_{p+2}=\cdots=\xi$. Furthermore, $T_{h+k} \xi=\xi$ for $k \geqq K(p, h)$.

Proof. Let $T_{h} x_{p}=x_{p}$. Then $T_{h} T_{p+1} x_{p}=T_{p+1} T_{h} x_{p}=T_{p+1} x_{p}$, i.e. $T_{h} x_{p+1}=x_{p+1}$. Assume $T_{h} x_{p+j}=x_{p+j}(j>1)$. Then

$$
T_{h} x_{p+j+1}=T_{h} T_{p+j+1} x_{p+j}=T_{p+j+1} T_{h} x_{p+j}=T_{p+j+1} x_{p+j}=x_{p+j+1},
$$

i.e. $T_{h} x_{p+q}=x_{p+q} ; q=0,1,2, \cdots$.

Hence $d\left(x_{p+q}, x_{p+q^{\prime}}\right)=d\left(T_{h} x_{p+q}, T_{h} x_{p+q^{\prime}}\right)$, contradicting (1), unless $x_{p+q}=x_{p+q^{\prime}} ;$ for, by $(4), d\left(x_{p+q}, x_{p+q^{\prime}}\right) \leqq C$. Therefore, all elements in the set $\left\{x_{p+q}\right\}_{q=0,1}, \ldots$ coincide. Call their common value $\xi$. Choose $K$ so large that $h+K=p+1$ (in case $h>p$, take $K=0$ ). Then, if $k$ $\geqq K: T_{h+k} \xi=T_{h+k} x_{h+k-1}=x_{h+k}=\xi$, and our Lemma is proved.

Proof of THE TheOREM. We will assume that no element of the set $\left\{x_{n}\right\}_{n \geq m}$ is fixed under any of the elements of $\left\{T_{k}\right\}_{k \geq m}$. Otherwise, by the Lemma, the first part of the theorem would be already proved (and even the second one, if $h=1$ and $K=0$ ).

Define $\rho_{i}(x, y)=d\left(T_{i} x, T_{i} y\right) / d(x, y)$, for $0<d(x, y) \leqq C$. Let $N$ be the index specified in (3). Without loss, we may assume $N \geqq m$. (If not, we take a suitable $N^{\prime}>N$.) 
Then $d\left(x_{N+1}, x_{N}\right) \leqq C$,

$$
\begin{aligned}
d\left(x_{N+2}, x_{N+1}\right) & =d\left(T_{N+2} x_{N+1}, x_{N+1}\right)=d\left(T_{N+2} T_{N+1} x_{N}, T_{N+1} x_{N}\right) \\
& =d\left(T_{N+1} T_{N+2} x_{N}, T_{N+1} x_{N}\right) \\
& =\rho_{N+1}\left(T_{N+2} x_{N}, x_{N}\right) d\left(T_{N+2} x_{N}, x_{N}\right) \\
& \leqq C \rho_{N+1}\left(T_{N+2} x_{N}, x_{N}\right)
\end{aligned}
$$

where we used (2), (3a) and the definition of $\rho_{i}(x, y)$.

Using the same arguments

$$
\begin{aligned}
d\left(x_{N+3}, x_{N+2}\right) & =d\left(T_{N+3} x_{N+2}, x_{N+2}\right)=d\left(T_{N+3} T_{N+2} x_{N+1}, T_{N+2} x_{N+1}\right) \\
& =d\left(T_{N+2} T_{N+3} x_{N+1}, T_{N+2} x_{N+1}\right) \\
& =\rho_{N+2}\left(T_{N+3} x_{N+1}, x_{N+1}\right) d\left(T_{N+3} x_{N+1}, x_{N+1}\right) \\
& =\rho_{N+2}\left(T_{N+3} x_{N+1}, x_{N+1}\right) d\left(T_{N+3} T_{N+1} x_{N}, T_{N+1} x_{N}\right) \\
& =\rho_{N+2}\left(T_{N+3} x_{N+1}, x_{N+1}\right) d\left(T_{N+1} T_{N+3} x_{N}, T_{N+1} x_{N}\right) \\
& =\rho_{N+2}\left(T_{N+3} x_{N+1}, x_{N+1}\right) \rho_{N+1}\left(T_{N+3} x_{N}, x_{N}\right) d\left(T_{N+3} x_{N}, x_{N}\right) \\
& \leqq C \rho_{N+2}\left(T_{N+3} x_{N+1}, x_{N+1}\right) \rho_{N+1}\left(T_{N+3} x_{N}, x_{N}\right) .
\end{aligned}
$$

And, in general

$$
\begin{aligned}
d\left(x_{N+k+1}, x_{N+k}\right) & \leqq C \prod_{i=0}^{k-1} \rho_{N+i+1}\left(T_{N+k+1} x_{N+i}, x_{N+i}\right) \\
& \leqq C \prod_{i=0}^{k-1} \rho_{N+1}\left(T_{N+k+1} x_{N}, x_{N}\right) \\
& =C\left[\rho_{N+1}\left(T_{N+k+1} x_{N}, x_{N}\right)\right]^{k} \\
& \leqq C\left[\rho_{N+1}\left(T_{N+1} x_{N}, x_{N}\right)\right]^{k}
\end{aligned}
$$

where we used $(3 b)$ and $(3 c)$. (We note that $\rho_{i}(x, y)$ is well defined for all elements in the sequence, because of the assumption made at the beginning of the proof.)

Now, let $n \geqq N$, say $n=N+q$. Then

$$
\begin{aligned}
d\left(x_{n+p}, x_{n}\right) & \leqq \sum_{k=0}^{p-1} d\left(x_{n+k+1}, x_{n+k}\right)=\sum_{k=0}^{p-1} d\left(x_{N+q+k+1}, x_{N+q+k}\right) \\
& \leqq \sum_{k=0}^{p-1} C\left[\rho_{N+1}\left(T_{N+1} x_{N}, x_{N}\right)\right]^{q+k} \\
& \leqq C\left[\rho_{N+1}\left(T_{N+1} x_{N}, x_{N}\right)\right]^{n-N} /\left(1-\rho_{N+1}\left(T_{N+1} x_{N}, x_{N}\right)\right)
\end{aligned}
$$

for, by definition, $0 \leqq \rho_{i}(x, y)<1,(i \geqq m)$.

Hence, $\left\{x_{n}\right\}$ is a Cauchy sequence. Therefore, there exists a $\xi \in X$ such that $x_{n} \rightarrow \xi$ as $n \rightarrow \infty$. Also 


$$
\begin{aligned}
d\left(T_{n} \xi, \xi\right) & \leqq d\left(T_{n} \xi, x_{n}\right)+d\left(x_{n}, \xi\right) \\
& =d\left(T_{n} \xi, T_{n} x_{n-1}\right)+d\left(x_{n}, \xi\right) \\
& <d\left(\xi, x_{n-1}\right)+d\left(x_{n}, \xi\right),
\end{aligned}
$$

since for $n>\nu, d\left(x_{n-1}, \xi\right) \leqq C$.

Therefore, $T_{n} \xi \rightarrow \xi$ as $n \rightarrow \infty$, and we have the first part of our theorem.

From (\#), on making $p \rightarrow \infty$, we get an estimate of the rate of convergence of $\left\{x_{n}\right\}$, namely

$$
d\left(x_{n}, \xi\right) \leqq C\left[\rho_{N+1}\left(T_{N+1} x_{N}, x_{N}\right)\right]^{n-N} /\left(1-\rho_{N+1}\left(T_{N+1} x_{N}, x_{N}\right)\right) .
$$

Assume now (5) holds. Hence $d\left(T_{h} T_{i} \xi, T_{i} \xi\right)=d\left(T_{h+i} \xi, T_{i} \xi\right)$. If we let $i \rightarrow \infty$, since $T_{h}$ is continuous, the first member tends to $d\left(T_{h} \xi, \xi\right)$, while the second one tends to zero. Hence, $T_{h} \xi=\xi$.

-Assume $T_{i} \eta \rightarrow \eta$ as $i \rightarrow \infty$. Then, as before, $T_{h} \eta=\eta$. Furthermore, by (4) $d(\xi, \eta) \leqq C$. If $\xi \neq \eta$, we would have $d(\xi, \eta)=d\left(T_{h} \xi, T_{h} \eta\right)$, contradicting (1), for $h \geqq m$. Hence, $d(\xi, \eta)=0$ and $\xi$ is unique.

Since $T_{i} \xi=T_{i} T_{h} \xi=T_{h} T_{i} \xi$, and $\xi$ is a unique fixed point of $T_{h}$, we have $T_{i} \xi=\xi(i=1,2, \cdots)$ concluding thus the proof of our theorem.

Finally, we give three examples to illustrate the extents and limitations of our results. The first one is a direct application of Theorem 2 , for which no previous results can be used. The second one shows that condition ( $3 \mathrm{~b})$ is by no means necessary. To counterballast this, the last example shows a transformation without fixed points, which does not fulfill requirement $(3 \mathrm{~b})$.

EXAMPLE 2. Let $X=\left\{x=e^{i \theta} / 0 \leqq \theta \leqq 3 \pi / 2\right\}, \quad d(x, y)=|x-y|$ $=2\left|\sin \left(\left(\theta_{x}-\theta_{y}\right) / 2\right)\right|$.

Define $T: X \rightarrow X$ such that $T x=e^{i \theta_{x} / 3}$ (i.e. $T_{i} \equiv T$, all $i$ ). Let $0<C$ $<2^{1 / 2}$. Hence, it is clear that $d(T x, T y)<d(x, y)$ for $0<d(x, y) \leqq C$. (The inequality can not be improved, for $d(T x, T y) / d(x, y) \rightarrow 1$ when $x \rightarrow e^{i 0}, y \rightarrow e^{i 3 \pi / 2}$.)

Let $x_{0}=e^{i \theta} \in X$, arbitrary. Then, $x_{n}=T x_{n-1}=e^{i \theta / 3^{n}}=e^{i \theta_{n}}$. Since $d\left(x_{n+1}, x_{n}\right)=2\left|\sin \left(\left(\theta_{n+1}-\theta_{n}\right) / 2\right)\right|=2\left|\sin \left(\theta / 3^{n+1}\right)\right|$, it is clear that for $n \geqq N_{1}\left(x_{0}\right), d\left(x_{n+1}, x_{n}\right) \leqq C$, and we have (3a).

In order to show (3b), we observe that in this case the condition reduces to

$$
d\left(x_{n+3}, x_{n+2}\right) / d\left(x_{n+2}, x_{n+1}\right) \leqq d\left(x_{n+2}, x_{n+1}\right) / d\left(x_{n+1}, x_{n}\right)
$$

when $n \geqq N\left(x_{0}\right)$ and the denominators do not vanish. This last alternative occurs only when $\theta=0$, where the final result is trivial. So that we may assume $\theta \neq 0$. Let $\phi=\theta / 3^{n+3}$. With this notation, (3b) will hold if and only if 
$2 \sin \phi / 2 \sin 3 \phi \leqq 2 \sin 3 \phi / 2 \sin 9 \phi$.

Clearly, taking $n \geqq N_{2}\left(x_{0}\right)$, we will have $0 \leqq 9 \phi \leqq \pi / 2$.

Consider the function $f(t)=\sin 3 t / \sin t=2 \cos ^{2} t+\cos 2 t, 0<t \leqq \pi / 2$. A simple computation shows $f^{\prime}(t)<0$. Hence, $f(t)$ decreases, and $\sin t / \sin 3 t$ increases with $t$. In particular $\sin \phi / \sin 3 \phi<\sin 3 \phi / \sin 9 \phi$, which is precisely what we wanted to prove.

As stated in Remark (a), (3c) is obviously satisfied.

Also, condition (4) is immediate.

Hence, we can apply our Theorem and conclude: $x_{n} \rightarrow \xi=e^{i_{0}}$ as $n \rightarrow \infty$, and $T \xi=\xi$.

We observe that neither Theorem 1 nor Theorem 3 in [5] can be used in this case, whereas Theorem 2 in [5] asserts at best the periodicity of $\xi$.

EXAmpLE 3. Let $X$ be the nonnegative reals with the Euclidean metric. Define $T: X \rightarrow X$ by $T x=x /(x+1)$. It is easy to show that $d(T x, T y)<d(x, y)$ whenever $x \neq y$, so that $C>0$ can be chosen arbitrarily.

Let $x_{0} \in X, x_{0} \neq 0, x_{n}=T x_{n-1}$. (The case $x_{0}=0$ is trivial.) Hence $x_{n}=x_{0}\left(n x_{0}+1\right)^{-1}$ and

$$
d\left(x_{n+1}, x_{n}\right)=x_{0}^{2} /\left[(n+1) x_{0}+1\right]\left(n x_{0}+1\right) .
$$

Therefore

$$
\frac{d\left(x_{n+3}, x_{n+2}\right)}{d\left(x_{n+2}, x_{n+1}\right)}-\frac{d\left(x_{n+2}, x_{n+1}\right)}{d\left(x_{n+1}, x_{n}\right)}=\frac{2 x_{0}^{2}}{\left[(n+3) x_{0}+1\right]\left[(n+2) x_{0}+1\right]}>0
$$

and hence $(3 \mathrm{~b})$ is not satisfied. Nevertheless, $x_{n} \rightarrow 0 \in X$ as $n \rightarrow \infty$, $T 0=0$, and 0 is the only element with this property.

EXAmple 4. We will use Rakotch's example mentioned at the beginning of this Section.

Let $X$ be the nonnegative reals with the Euclidean metric, and $T x=\ln \left(1+e^{x}\right)$. As shown there $d(T x, T y)<d(x, y)$ whenever $x \neq y$, so that $C>0$ can be chosen arbitrarily. Let $x_{0} \in X, x_{0} \neq 0$, and $x_{n}=T x_{n-1}$. (The case $x_{0}=0$ is trivial.) Hence, as shown by induction $x_{n}=\ln \left(n+e^{x_{0}}\right)$. Since $d\left(x_{n+1}, x_{n}\right)=\ln \left(1+1 /\left(n+e^{x_{0}}\right)\right)$, we see that for $n \geqq N\left(x_{0}\right), d\left(x_{n+1}, x_{n}\right) \leqq C$. Let $f(t)=(\ln ((t+2) /(t+1)) /(\ln (t+1) / t))$, $t>1$. Clearly, $f(t)<1$, and $f(t) \rightarrow 1$ as $t \rightarrow \infty$.

Also,

$$
\begin{aligned}
f^{\prime}(t)= & {\left[(t+1) \ln ^{2}((t+1) / t)\right]^{-1} } \\
\cdot & {[(1 / t) \ln ((t+2) /(t+1))-(1 /(t+2)) \ln ((t+1) / t)] . }
\end{aligned}
$$


Assume that for some $s>1, f^{\prime}(s)=0$. Hence, we have the following chain of equivalences.

$$
\begin{aligned}
f^{\prime}(s) & =0 \Leftrightarrow \ln ((s+2) /(s+1))^{s+2} \\
& =\ln ((s+1) / s)^{s} \Leftrightarrow(1+1 /(s+1))^{s+2}=(1+1 / s)^{s} .
\end{aligned}
$$

Therefore $(1+1 / s)^{s}>(1+1 /(s+1))^{s+1}$, which is clearly impossible, for the function $(1+1 / t)^{t}$ increases with $t$. Therefore, $f^{\prime}(t)$ has constant sign. If it were $f^{\prime}(t)<0$, then $f(t)$ would be a decreasing function, and since $f(t) \rightarrow 1$ when $t \rightarrow \infty$, we would have $f(t) \geqq 1$, which is impossible. Therefore, $f(t)$ is increasing. In particular: $f\left(n+1+e^{x_{0}}\right)$ $>f\left(n+e^{x_{0}}\right)$, i.e. (3b) does not hold.

It is obvious that $x_{n} \rightarrow \infty \notin X, T x_{n} \rightarrow \infty$ as $n \rightarrow \infty$, and furthermore $T$ does not have any fixed points in $X$.

\section{REFERENCES}

1. A. F. Monna, Sur un Theorème de M. Luxemburg concernant les points fixes d'une classe d'applications d'un espace metrique dans lui même, Akad. van Wet. Amst. Proc. Ser. A 64 (1961), 89-96.

2. W. A. J. Luxemburg, On the convergence of successive approximations in the theory of ordinary differential equations. II, Nieuw archief voor wiskunde (3) 6(1958), 93-98.

3. M. Edelstein, $A$ remark on a theorem of A. F. Monna, Akad. van Wet. Amst. Proc. Ser. A 67 (1964), 88-89.

4. E. Rakotch, $A$ note on contractive mappings, Proc. Amer. Math. Soc. 13 (1962), 459-465.

5. M. Edelstein, On fixed and periodic points under contractive mappings, J. London Math. Soc. 37 (1962), 74-79.

Universidad Nacional de la Plata, Argentina 